\title{
Generalizations of Heilbronn's Triangle Problem
}

\author{
Hanno Lefmann ${ }^{1}$ \\ Fakultät für Informatik, TU Chemnitz, D-09107 Chemnitz, Germany
}

\begin{abstract}
For integers $d, j \geq 2$ and $n \geq j$, distributions of $n$ points in the $d$-dimensional unit cube $[0,1]^{d}$ are investigated, such that the minimum volume of the convex hull determined by $j$ of $n$ points is large. Lower and upper bounds on these minimum volumes are given. For obtaining lower bounds, results on the independence number of non-uniform, linear hypergraphs are used, which might be of interest by their own.
\end{abstract}

Keywords: Hypergraphs, Independence Number, Large Simplices.

\section{Introduction}

Originally, Heilbronn's triangle problem asks for the supremum value $\Delta_{3}(n)$, over all distributions of $n$ points in the unit square $[0,1]^{2}$, of the mininum area of a triangle among $n$ points. For prime numbers $n$, the points $(1 / n)$. $\left(i \bmod n, i^{2} \bmod n\right), i=0, \ldots, n-1$, yield $\Delta_{3}(n)=\Omega\left(1 / n^{2}\right)$ as has been observed by Erdös. By using results on the independence number of certain 3-uniform hypergraphs, this lower bound has been improved by Komlós, Pintz and Szemerédi [8] to $\Delta_{3}(n)=\Omega\left(\log n / n^{2}\right)$. Earlier upper bounds on $\Delta_{3}(n)$ have been proved by Roth and Schmidt, and the currently best upper bound

1 Email: lefmann@informatik.tu-chemnitz.de 
$\Delta_{3}(n)=O\left(2^{c \sqrt{\log n}} / n^{8 / 7}\right)$ is due to Komlós, Pintz and Szemerédi [7]. A variant of this problem, considered by Schmidt [10], asks for integers $2 \leq j \leq n$ for the supremum value $\Delta_{j}(n)$, over all distributions of $n$ points in $[0,1]^{2}$, of the mininum area of the convex hull of $j$ among $n$ points, and for $j=4$ he obtained $\Delta_{4}(n)=\Omega\left(1 / n^{3 / 2}\right)$. For fixed $j \geq 3$, the currently best lower bound $\Delta_{j}(n)=\Omega\left((\log n)^{1 /(j-2)} / n^{(j-1) /(j-2)}\right)$ is due to this author. However, for fixed $j \geq 4$ only the upper bound $\Delta_{j}(n)=O(1 / n)$ is known.

An extension of Heilbronn's triangle problem to higher dimensions has been considered by Barequet and Naor [2,3]. For integers $d, j, n \geq 2$, let $\Delta_{j, d}(n)$ be the supremum, over all distributions of $n$ points in the unit cube $[0,1]^{d}$, of the minimum $((j-1)$-dimensional for $j \leq d+1)$ volume of a $j$-point simplex among $n$ points. For fixed $3 \leq j \leq d+1$, the currently best bounds are $\Delta_{j, d}(n)=\Omega\left((\log n)^{1 /(d-j+2)} / n^{(j-1) /(d-j+2)}\right)$ and $\Delta_{j, d}(n)=O\left(n^{(j-1) / d}\right)$, moreover $\Delta_{j, d}(n)=O\left(n^{((j-1) / d))+(j-2) /(2 d(d-1))}\right)$ for even $j \geq 4$, see [9].

In connection with some range searching problems Chazelle [5] has investigated the function $\Delta_{j, d}(n)$ for values $j$, which depend on $n$, and he showed the asymptotically correct order $\Theta(j / n)$ with $\log n \leq j \leq n$ and fixed $d \geq 2$. In view of these results, it might be of interest, whether for a given range of $j$, say $3 \leq j \leq K$, there is a single configuration of $n$ points in $[0,1]^{d}$ which yields good lower bounds on the minimum volume of any $j$ points among the $n$ points, simultaneously for $j=3, \ldots, K$. To do so, we consider an extension of a result of Ajtai, Komlós, Pintz, Spencer and Szemerédi [1] on the independence number of hypergraphs without short cycles. Some of the probabilistic existence arguments, which are used in the proofs, can be derandomized in suitable discrete lattices and yield deterministic polynomial time algorithms.

\section{Uncrowded and Linear Hypergraphs}

Definition 2.1 For a hypergraph $\mathcal{G}$ the notation $\mathcal{G}=\left(V, \mathcal{E}_{2} \cup \ldots \cup \mathcal{E}_{k}\right)$ means that $V$ is its vertex-set and $\mathcal{E}_{i}$ is the set of all $i$-element edges in $\mathcal{G}, i=2, \ldots, k$. The quantity $i \cdot\left|\mathcal{E}_{i}\right| /|V|$, denoted by $t_{i}^{i-1}$, is the average degree of a vertex for the $i$-element edges $E \in \mathcal{E}_{i}$ in $\mathcal{G}$. A hypergraph $\mathcal{G}=\left(V, \mathcal{E}_{k}\right)$ is called $k$-uniform, i.e., each edge $E \in \mathcal{E}$ contains exactly $k$ vertices. The independence number $\alpha(\mathcal{G})$ of a hypergraph $\mathcal{G}=(V, \mathcal{E})$ is the largest size of a subset $I \subseteq V$ which contains no edges from $\mathcal{E}$. A $j$-cycle in $\mathcal{G}=(V, \mathcal{E})$ is a sequence $E_{1}, \ldots, E_{j}$ of distinct edges from $\mathcal{E}$, such that $E_{i} \cap E_{i+1} \neq \emptyset, i=1, \ldots, j-1$, and $E_{j} \cap E_{1} \neq \emptyset$, and a sequence $v_{1}, \ldots, v_{j}$ of distinct vertices with $v_{i+1} \in E_{i} \cap E_{i+1}, i=1, \ldots, j-1$, and $v_{1} \in E_{1} \cap E_{j}$. An unordered pair $\left\{E, E^{\prime}\right\}$ of distinct edges $E, E^{\prime} \in \mathcal{E}$ with $\left|E \cap E^{\prime}\right| \geq 2$ is called 2-cycle. A 2-cycle $\left\{E, E^{\prime}\right\}$ in $\mathcal{G}=\left(V, \mathcal{E}_{2} \cup \ldots \cup \mathcal{E}_{k}\right)$ with 
$E \in \mathcal{E}_{i}$ and $E^{\prime} \in \mathcal{E}_{j}$ is called $(2 ;(g, i, j))$-cycle iff $\left|E \cap E^{\prime}\right|=g, 2 \leq g \leq i \leq j$ but $g<j$. The hypergraph $\mathcal{G}$ is called linear if it does not contain any 2-cycles, and it is called uncrowded if it does not contain any 2-, 3-, or 4-cycles.

Well known is Turán's theorem for hypergraphs:

Theorem 2.2 Let $\mathcal{G}=\left(V, \mathcal{E}_{2} \cup \ldots \cup \mathcal{E}_{k}\right)$ be a hypergraph on $|V|=N$ vertices and with average degree $t_{i}^{i-1}:=i \cdot\left|\mathcal{E}_{i}\right| / N$ for the $i$-element edges, $i=2, \ldots, k$. Let $t_{i_{0}}:=\max \left\{t_{i} \mid 2 \leq i \leq k\right\} \geq 1 / 2$. Then, there exists an independent set $I \subseteq V$ in $\mathcal{G}$ with $|I|=\Omega\left(N / t_{i_{0}}\right)$.

The next result by Ajtai, Komlós, Pintz, Spencer and Szemerédi [1] improves on Theorem 2.2 for uncrowded, uniform hypergraphs, compare [4] for a deterministic polynomial time algorithm.

Theorem 2.3 Let $k \geq 3$ be fixed. Let $\mathcal{G}=\left(V, \mathcal{E}_{k}\right)$ be an uncrowded, $k$-uniform hypergraph on $|V|=N$ vertices and with average degree $t_{k}^{k-1}:=k \cdot\left|\mathcal{E}_{k}\right| / N$. Then, the independence number $\alpha(\mathcal{G})$ satisfies $\alpha(\mathcal{G})=\Omega\left((N / t) \cdot\left(\log t_{k}\right)^{1 /(k-1)}\right)$.

Theorem 2.3 can be extended to non-uniform hypergraphs as follows:

Theorem 2.4 Let $k \geq 3$ be fixed. Let $\mathcal{G}=\left(V, \mathcal{E}_{3} \cup \ldots \cup \mathcal{E}_{k}\right)$ be an uncrowded hypergraph on $|V|=N$ vertices, such that for some number $T \geq 1$ for $i=$ $3, \ldots, k$ the average degrees $t_{i}^{i-1}:=i \cdot\left|\mathcal{E}_{i}\right| / N$ for the $i$-element edges satisfy $t_{i}^{i-1} \leq T^{i-1} \cdot(\log T)^{(k-i) /(k-1)}$. Then, the independence number $\alpha(\mathcal{G})$ satisfies $\alpha(\mathcal{G})=\Omega\left((N / T) \cdot(\log T)^{1 /(k-1)}\right)$.

For a certain range $k<T<N$ of the parameters Theorem 2.4 is asymptotically best possible up to a constant factor, as can be seen by considering random non-uniform hypergraphs $\mathcal{G}=\left(V, \mathcal{E}_{3} \cup \ldots \cup \mathcal{E}_{k}\right)$ on $|V|=N$ vertices. Theorem 2.4 also holds for linear hypergraphs, compare [6] for the case of uniform hypergraphs:

Theorem 2.5 Let $k \geq 3$ be fixed. Let $\mathcal{G}=\left(V, \mathcal{E}_{3} \cup \ldots \cup \mathcal{E}_{k}\right)$ be a linear hypergraph with $|V|=N$, such that for some number $T \geq 1$ for $i=3, \ldots, K$ the average degrees $t_{i}^{i-1}:=i \cdot\left|\mathcal{E}_{i}\right| / N$ for the $i$-element edges satisfy $t_{i}^{i-1} \leq$ $T^{i-1} \cdot(\log T)^{(k-i) /(k-1)}$. Then, there exists an independent set $I \subseteq V$ with $|I|=\Omega\left((N / T) \cdot(\log T)^{1 /(k-1)}\right)$.

With Theorem 2.5 the following has been shown recently by this author:

Theorem 2.6 Let $d \geq 2$ and $K \geq 3$ be fixed. For integers $n \geq K$ there exist $n$ points in $[0,1]^{2}$, such that, simultaneously for $j=3, \ldots, K$, the area of the convex hull of any $j$ among the $n$ points is $\Omega\left((\log n)^{1 /(j-2)} / n^{(j-1) /(j-2)}\right)$. 


\section{Volumes of the Convex Hull of $\mathrm{j}$ Points}

In view of Theorem 2.6 we turn our attention to distributions of $n$ points in higher dimensional unit cubes. A simplex given by $j$ points $P_{1}, \ldots, P_{j} \in[0,1]^{d}$, where $j \leq d+1$, is the set of all points $P_{1}+\sum_{i=2}^{j} \lambda_{i} \cdot\left(P_{i}-P_{1}\right)$ with $\lambda_{i} \geq 0$, $i=2, \ldots, j$, and $\sum_{i=2}^{j} \lambda_{i} \leq 1$. The $((j-1)$-dimensional $)$ volume of a simplex determined by points $P_{1}, \ldots, P_{j} \in[0,1]^{d}, 2 \leq j \leq d+1$, is vol $\left(P_{1}, \ldots, P_{j}\right):=$ $(1 /(j-1) !) \cdot \prod_{i=2}^{j} \operatorname{dist}\left(P_{i} ;\left\langle P_{1}, \ldots, P_{i-1}\right\rangle\right)$, where dist $\left(P_{i} ;\left\langle P_{1}, \ldots, P_{i-1}\right\rangle\right)$ is the Euclidean distance of the point $P_{i}$ from the affine space $\left\langle P_{1}, \ldots, P_{i-1}\right\rangle$ generated by the points $P_{1}, \ldots, P_{i-1}$ with $\left\langle P_{1}\right\rangle:=P_{1}$.

By using Theorem 2.2 and, for an algorithmic version, results on the theory of lattices, the following has been shown by this author:

Theorem 3.1 Let $K, d \geq 3$ be fixed. For every $n \geq K$ there exists a configuration of $n$ points in the unit cube $[0,1]^{d}$ such that, simultaneously for $j=2, \ldots, K$, the volume of the convex hull of any $j$ among the $n$ points is $\Omega\left(1 / n^{(j-1) /(1+|d-j+1|)}\right)$.

By Theorem 3.1 we obtain a single distribution of $n$ points in $[0,1]^{d}$, which is simultaneously good for small sets of points, however for $3 \leq j \leq d+1$ in view of the best available lower bounds we loose a polylogarithmic factor. The next result takes care of this factor.

Theorem 3.2 Let $d \geq 2$ and $K \geq 3$ with $K \leq d+1$ be fixed. For every $n \geq K$ there exists a configuration of $n$ points in the unit cube $[0,1]^{d}$, such that, simultaneously for $j=3, \ldots, K$, the $(j-1)$-dimensional volume of any $j$-point simplex among these $n$ points is $\Omega\left((\log n)^{1 /(d-j+2)} / n^{(j-1) /(d-j+2)}\right)$.

Proof (Sketch) Let $\beta, \gamma_{2}, \ldots, \gamma_{K}, d_{3}, \ldots, d_{K}>0$ be suitable constants, and set $N:=n^{1+\beta}$, and $V_{j}:=d_{j} \cdot(\log n)^{1 /(d-j+2)} / n^{(j-1) /(d-j+2)}, j=3, \ldots, K$. Uniformly at random and independently of each other select $N$ points $P_{1}, \ldots, P_{N} \in$ $[0,1]^{d}$. Form a hypergraph $\mathcal{G}=\left(V, \mathcal{E}_{2}^{*} \cup \mathcal{E}_{3}^{*} \cup \mathcal{E}_{3} \cup \cdots \cup \mathcal{E}_{K}^{*} \cup \mathcal{E}_{K}\right)$, where $\mathcal{E}_{j}^{*}, \mathcal{E}_{j} \subseteq[V]^{j}, j=2, \ldots, K$, with vertex-set $V=\left\{P_{1}, \ldots, P_{N}\right\}$ and with two types of $j$-element edges. For $j=2, \ldots, K$, and points $P_{i_{1}}, \ldots, P_{i_{j}}$, $1 \leq i_{1}<\ldots<i_{j} \leq N$, let $\left\{P_{i_{1}}, \ldots, P_{i_{j}}\right\} \in \mathcal{E}_{j}^{*}$ iff vol $\left(P_{i_{1}}, \ldots, P_{i_{j}}\right) \leq N^{-\gamma_{j}}$. Let $E=\left\{P_{i_{1}}, \ldots, P_{i_{j}}\right\} \in \mathcal{E}_{j}$ iff vol $\left(P_{i_{1}}, \ldots, P_{i_{j}}\right) \leq V_{j}$ and $E^{\prime} \nsubseteq E$ for all edges $E^{\prime} \in \mathcal{E}_{2}^{*} \cup \cdots \cup E_{j-1}^{*}$.

Let $C_{(g ; i, j)}$ be the set of all $(2 ;(g ;, i, j))$-cycles in the hypergraph $\mathcal{G}, 2 \leq$ $g \leq i \leq j \leq K$ and $g<j$. Set $\gamma_{j}:=(j-1) /(d-j+3 / 2), j=2, \ldots, K$, and $\beta:=1 /(5 \cdot d \cdot K)$. One can show that there exists a hypergraph $\mathcal{G}=$ $\left(V, \mathcal{E}_{2}^{*} \cup \mathcal{E}_{3}^{*} \cup \mathcal{E}_{3} \cup \cdots \cup \mathcal{E}_{K}^{*} \cup \mathcal{E}_{K}\right)$, which satisfies for constants $c_{j}>0$ : 


$$
\left|\mathcal{E}_{j}^{*}\right|=o(|V|) \quad \text { and } \quad\left|C_{(g ; i, j)}\right|=o(|V|) \quad \text { and } \quad\left|\mathcal{E}_{j}\right| \leq c_{j} \cdot N^{j} \cdot V_{j}^{d-j+2} \text {. }
$$

Delete one vertex from each edge $E \in \mathcal{E}_{2}^{*} \cup \cdots \cup \mathcal{E}_{K}^{*}$ and each $(2 ;(g, i, j))$ cycle in $C_{(g ; i, j)}, 2 \leq g \leq i \leq j \leq K$ with $g<j$, and obtain an induced, linear subhypergraph $\mathcal{G}^{0}=\left(V^{0}, \mathcal{E}_{3}^{0} \cup \cdots \cup \mathcal{E}_{k}^{0}\right)$ of $\mathcal{G}$ with $\mathcal{E}_{j}^{0}:=\mathcal{E}_{j} \cap\left[V^{0}\right]^{j}, j=3, \ldots, K$, on $\left|V^{0}\right|=(1-o(1)) \cdot N$ vertices. With $T:=c^{\prime} \cdot(N / n) \cdot(\log n)^{1 /(k-1)}$ for a suitable constant $c^{\prime}>0$ all assumptions in Theorem 2.5 are fulfilled and we infer for the independence number $\alpha\left(\mathcal{G}^{0}\right) \geq n$. Thus, there exist $n$ points such that the volume of every $j$-point simplex, $j=3, \ldots, K$, among these $n$ points is $\Omega\left((\log n)^{1 /(d-j+2)} / n^{(j-1) /(d-j+2)}\right)$ as claimed.

\section{References}

[1] Ajtai, M., Komlós, J., Pintz, J., Spencer, J., and Szemerédi, E., Extremal Uncrowded Hypergraphs, J. Comb. Theory Ser. A 32 (1982), 321-335.

[2] Barequet, G., A Lower Bound for Heilbronn's Triangle Problem in d Dimensions, SIAM J. Disc. Math. 14 (2001), 230-236.

[3] Barequet, G., and Naor, J., Large $k-D$ Simplices in the D-Dimensional Unit Cube, Proc. "17th Canadian Conf. on Computational Geometry CCCG'05" (2005), 31-34. URL: http://www.cccg.ca/proceedings/2005/27.ps

[4] Bertram-Kretzberg, C., and Lefmann, H., The Algorithmic Aspects of Uncrowded Hypergraphs, SIAM J. Computing 29 (1999), 201-230.

[5] Chazelle, B., Lower Bounds on the Complexity of Polytope Range Searching, J. Amer. Math. Soc. 2 (1989), 637-666.

[6] Duke, R. A., Lefmann, H., and Rödl, V., On Uncrowded Hypergraphs, Rand. Struct. \& Algorithms 6 (1995), 209-212.

[7] Komlós, J., Pintz, J., and Szemerédi, E., On Heilbronn's Triangle Problem, J. London Math. Soc. 24 (1981), 385-396.

[8] Komlós, J., Pintz, J., and Szemerédi, E., A Lower Bound for Heilbronn's Problem, J. London Math. Soc. 25 (1982), 13-24.

[9] Lefmann, H., Distributions of Points in $d$ Dimensions and Large $k$ Points Simplices, Proc. "11th Ann. Computing and Combinatorics Conf. COCOON'05", Springer, LNCS 3595, ed. Wang, L., (2005), 514-523.

[10] Schmidt, W. M., On a Problem of Heilbronn, J. London Math. Soc. (2) 4 (1972), $545-550$. 